\title{
Partially Coherent Optical Diffraction Tomography for Label-Free Quantitative Microscopy
}

\author{
Juan M. Soto ${ }^{1}$, José A. Rodrigo , Tatiana Alieva \\ Facultad de Ciencias Físicas, Universidad Complutense de Madrid \\ Ciudad Universitaria $s / n$, Madrid 28040, Spain \\ 1 juansoto@ucm.es
}

\begin{abstract}
In this work we present a tomographic technique for label-free quantitative microscopy that exploits the benefits of the partially coherent illumination. In contrast to conventional holographic techniques based on the coherent optical diffraction tomography (C-ODT), our approach uses an inexpensive noninterferometric setup comprising an electrically tunable lens and low spatially coherent illumination. This setup provides the refocusing needed for axially scan the sample and eventually producing a 3D stack of intensity images. Moreover, the partially coherent illumination avoids speckle noise, which plagues the $\mathrm{C}$ ODT imaging methods. This technique has been developed for optically weak and almost non-absorbing objects that meet the conditions of the first Born approximation. We experimentally demonstrate the capability of the proposed technique by recovering the 3D refractive index distribution of biological samples such as blood cells and diatoms. The results are similar to those obtained by the C-ODT but using a simplified reconstruction process and a refocusing module that can be easily included in commercial widefield microscopes.
\end{abstract}

\section{INTRODUCTION}

Label-free quantitative microscopy is an attractive alternative to staining techniques (e.g. fluorescence) often applied for different cell studies. Indeed, the refractive index (RI) behaves like a natural biomarker revealing relevant information (shape, density, volume, chemical composition). Most of the successful coherent optical diffraction tomography (C-ODT) proposals are based on digital holography using coherent illumination sources (e.g. laser). Particularly, in [1] a time multiplexing scheme that requires rotated beam illumination is implemented for 3D quantitative imaging with high spatial resolution. Nevertheless, the large amount of data to be acquired (at least one hologram for each position of the rotating illumination) and processed for assembling the synthetic 3D aperture in the frequency domain is the main bottleneck of this methodology. For speeding up the reconstruction, in [2] a spiral scanning of the illumination angles that minimizes the number of measurements has been proposed. A different approach considered here is using the partially coherent illumination that, in the same way as C-ODT, allows for the object RI recovering but also providing the following advantages: simplified data acquisition and reconstruction together with speckle-noise supression. Indeed, by using an illumination with low spatial coherence, the widefield microscope provides the same object frequency content as the C-ODT without needing beam rotation. However, in this case at least one stack of axially scanned intensity images is required because the numerical refocusing can not be used. Consequently, in this work we take advantage of the optical refocusing provided by an electrically tunable lens that can be easily incorporated in commercial microscopes [3]. This focus tunable lens also allows for accelerating and automating the RI reconstruction.

\section{FUNDAMENTALS OF 3D PARTIALLY COHERENT QUANTITATIVE IMAGING}

For a proper RI reconstruction, let us first introduce the complex scattering potential function as $V(\mathbf{r})=k_{0}^{2}\left[n_{s}^{2}(\mathbf{r})-n_{m}^{2}\right]$, where $\mathbf{r}=(x, y, z), k_{0}$ is the light wavenumber whereas $n_{s}(\mathbf{r})$ and $n_{m}$ are the RI of the specimen and its surrounding medium, respectively. $V(\mathbf{r})$ can be separated into real (phase $P(\mathbf{r})$ ) and imaginary (absorption $A(\boldsymbol{r})$ ) terms: $V(\mathbf{r})=P(\mathbf{r})+\mathrm{i} A(\mathbf{r})$. Thus, $A(\mathbf{r})=0$ for purely phase (nonabsorbing) samples. Following [4] the 3D intensity images (stack of refocused images) recorded by an optical system can be written in the frequency domain as $\left(\boldsymbol{\rho}=\left(\rho_{x}, \rho_{y}, \rho_{z}\right)\right)$ as

$$
\widehat{I}(\boldsymbol{\rho})=B \cdot \delta(\boldsymbol{\rho})+\widehat{P}(\boldsymbol{\rho}) \cdot H_{P}(\boldsymbol{\rho})+\widehat{A}(\boldsymbol{\rho}) \cdot H_{A}(\boldsymbol{\rho}),
$$

where $H_{P}$ and $H_{A}$ are the optical transfer functions (OTFs) of the phase and the absorption terms, correspondingly, and $B$ is the background intensity. The expression for $H_{P}$ and $H_{A}$, by assuming circular pupils and $n_{s}>n_{m}$, can be found in [4] for the paraxial case and in [5] for the nonparaxial context. As long as the sample is weak absorbing, the approximation $A(\boldsymbol{r})=\varepsilon \cdot P(\boldsymbol{r})$ (in which $\varepsilon=0-0.1$ ) is valid and the Eq.1 is rewritten as

$$
\hat{I}(\boldsymbol{\rho})=B \cdot \delta(\boldsymbol{\rho})+P(\boldsymbol{\rho}) \cdot\left[H_{P}(\boldsymbol{\rho})+\varepsilon \cdot H_{A}(\boldsymbol{\rho})\right] .
$$

Then, $P(\boldsymbol{\rho})$ is obtained by Wiener deconvolution [6]. Once $P(\boldsymbol{r})$ is calculated (by an inverse Fourier transform of $P(\boldsymbol{\rho})$ ), the $3 \mathrm{D}$ RI of the sample is computed by applying

$$
n_{s}^{2}(\boldsymbol{r})=P(\boldsymbol{r}) \cdot k_{0}^{-2}(1+\mathrm{i} \varepsilon)+n_{m}^{2} .
$$

Henceforth, only the real part of $n_{s}$ is considered as the absorption effect of the sample is negligible. The form of the OTFs strongly depends on the numerical aperture (NA) of the 


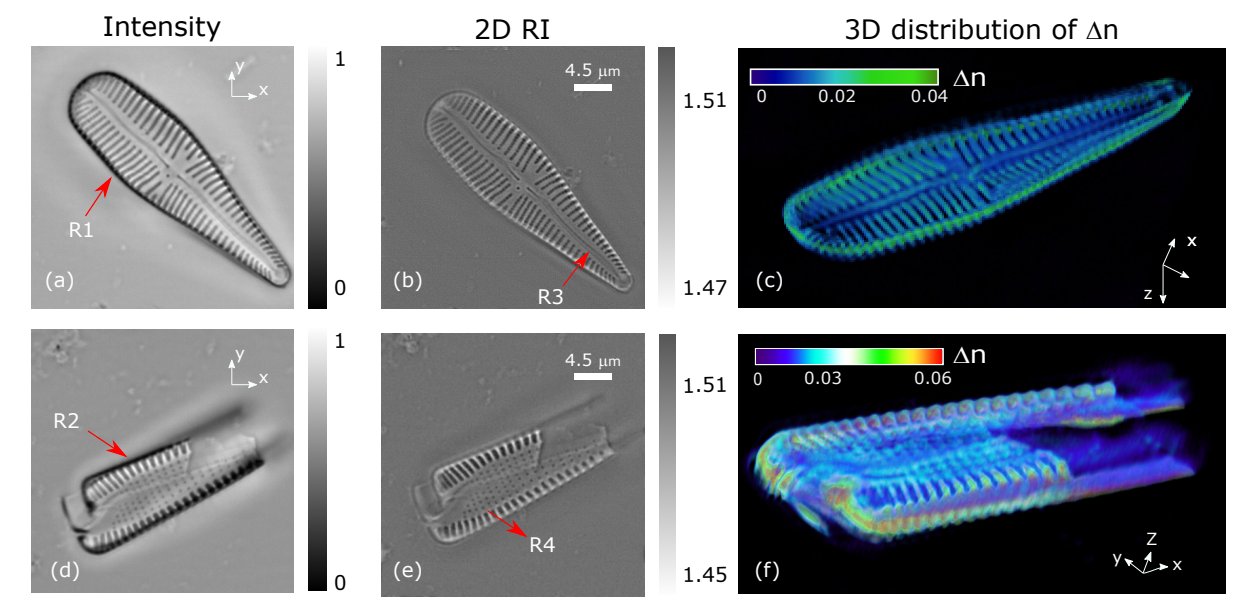

Fig. 1. 2D views within the XY-plane of the intensity (a-d) and RI (b-e) for two different diatom specimens immersed in oil $\left(n_{m}=1.51\right)$. (c-f) corresponds to the 3D distribution of $\Delta n$.

condenser $\left(\mathrm{NA}_{C}\right)$ and the objective $\left(\mathrm{NA}_{O}\right)$ of the microscope. For a fixed $\mathrm{NA}_{O}$, the spatial frequency content transmitted by the system is larger for higher $\mathrm{NA}_{C}$ (associated with a lower spatial coherence), thereby providing a better resolution and sectioning in the object reconstruction. Consequently, both high $\mathrm{NA}_{C}$ and $\mathrm{NA}_{O}$ are required for successful PCODT implementation. Further details regarding the PC-ODT technique are found in [3].

\section{EXPERIMENTAL SETUP}

First, the light coming from a quasi-monochromatic LED $\left(\lambda_{0}=450 \mathrm{~nm}\right)$ is collected by a high NA condenser lens $\left(\mathrm{NA}_{C}=0.95\right)$. Then, the sample is imaged with an objective lens $\left(\mathrm{NA}_{O}=1.4\right)$ and a tube lens. Both pupils (condenser and objective) are considered circular, henceforth. Next, the 3D image is axially scanned by using the refocusing module comprising a relay lens (RL) and an electrically tunable lens (Optotune, EL-10-30-C) which changes its focal length depending on the current it receives. Further details of this module are available in [7]. Then, the image is recorded by a high-speed sCMOS camera (Hamamatsu, Orca Flash 4.0). It is worth pointing out that the refocusing module plays a crucial role in the proposed framework. The mechanical refocusing consists of changing the distance between the stage of the sample and the objective lenses, thereby providing a constant NA for all refocused intensity slices. Conversely, the optical refocusing considered here allows for axial scanning of the sample without moving it. This method is faster and more precise than the mechanical stage control of the commercial microscopes. Nevertheless, such optical refocusing as well as the numerical refocusing usually applied in C-ODT produces a variation in the NA ( $\triangle \mathrm{NA})$ between different planes of the sample. In our case, the greatest thickness of the samples is around $5 \mu \mathrm{m}$ so that $\Delta \mathrm{NA} \sim 0.05 \cdot \mathrm{NA}_{O}$. This moderate $\Delta \mathrm{NA}$ justifies the shift-invariant OTF hypothesis (in which the whole theoretical background is supported) in the PC-ODT context.

\section{RESULTS AND DISCUSSION}

To test the performance of the PC-ODT technique, several samples (diatoms and blood cells) are considered. The diatoms are micro-organisms whose walls are made of silicon dioxide $\left(n_{\mathrm{SiO}_{2}} \sim 1.45-1.46\right.$ for the illumination wavelength $\left.\lambda_{0}\right)$. The usefulness of diatom analysis to validate the PC-ODT lies in the little size of the pores $(0.1-1 \mu \mathrm{m})$ they usually exhibit onto their walls. In this way, the reconstruction of such tiny features is a challenging test to the PC-OCT technique. Fig.1 shows slices of the intensity (Fig. 1a and Fig.1d) and the corresponding RI (Fig.1b and Fig.1e) distributions in the XY-plane for two different diatoms (immersed in oil: $n_{m}=$ 1.518 , Olympus type-F). Note that the bright-field intensity recorded by the camera suffers from some halos and a poor definition of the boundaries of the sample (see R1 and R2). These problems are mostly mitigated after the deconvolution procedure associated with the RI reconstruction, which in turn provides a deblurring of the central channel of the first diatom (see R3) along with a sharpening of the tiny pores (see R4) of the second one. The RI varies between $n_{s}=1.45-1.47$ which is in good agreement with those reported by [8]. Note that the RI is higher near the pores than on the solid walls of the diatom. The explanation of this lies in the porosity of the sample: if the immersion oil fills the holes, then the RI of the sample tends to the oil RI. The 3D RI distribution $\Delta n(\mathbf{r})=\left|\operatorname{Re}\left\{n_{s}(\mathbf{r})\right\}-n_{m}\right|$ for both diatoms is displayed in Fig. 1c and Fig.1f, correspondingly. Fiji 3D Volume Viewer [9] is used for visualization. It is worth pointing out that in this example $n_{s}(\mathbf{r})<n_{m}$, but the PC-ODT is also valid in the opposite case, as shown in Fig. 2 for another diatom immersed in silicone oil ( $n_{m}=1.406$, Immersol Sil 406, Zeiss). In the considered example, the wall of the diatom is around $n_{s}=$ $1.44-1.45$ (Fig.2b). Furthermore, the pores and other fine details of the diatom are revealed in the RI in comparison with the intensity stack.

Let us now discussing about the range of $|\Delta n|$ (linked to the phase contrast between the object and the medium) in 
(a) Intensity

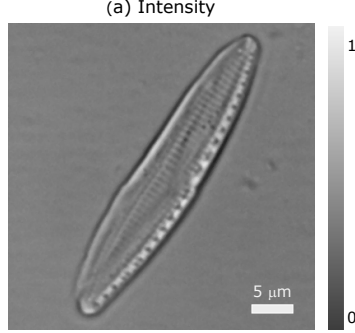

(b) Refractive index

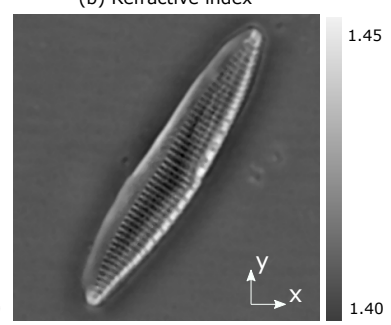

Fig. 2. 2D views within the XY-plane of the (a) intensity and (b) RI of a diatom sample immersed in silicone oil $\left(n_{m}=1.406\right)$.

which Born approximation works well. In the previous cases, the maximum $|\Delta n|$ is between $0.04-0.06$. A lower $|\Delta n|$ example is illustrated in Fig. 3 where glycerine $\left(n_{m}=1.473\right)$ is chosen as the immersion medium. As glycerine is close to $n_{\mathrm{SiO}_{2}} \sim 1.46$, the diatom is more transparent, thereby providing a worse contrast to the RI. Despite this, similar results are obtained for the silica wall $\left(n_{s} \sim 1.458\right)$ regardless the chosen surrounding medium. Note that the technique can not be applied for diatoms immersed in water $\left(n_{m}=1.33\right)$ due to the high RI contrast $(\Delta n \sim 0.12)$ beyond the weak object approximation.

In Fig.4 the reconstructed RI for a human blood smear is displayed, where red blood cells (RBCs) and white blood cells (WBCs) are observed. A RI range of $n_{R B C}=1.34-1.35$ is obtained for the RBC while $n_{W B C}=1.35-1.38$ for the WBCs (lymphocytes). These results indicate the feasibility of using PC-ODT as a diagnosis tool in biomedicine. Additionally, RI better reveals the real shape of the sample because bright-field images suffer from diffraction and OTF modulation. For example, the RI of RBCs provides not only valuable information about their shape and composition (e.g. hemoglobin concentration) but also allows the detection of parasites (see [10], where Plasmodium falciparum is found through a RI map analysis). Additionally, mutations of the forms of the diatoms provide information about the environment in which they live [11].
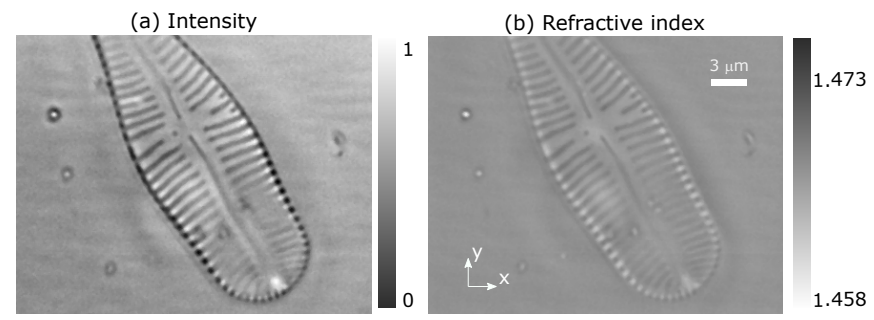

Fig. 3. 2D views within the XY-plane of the (a) intensity and (b) RI of a diatom immersed in glycerine $\left(n_{m}=1.473\right)$.

\section{COnClusions}

The aim of this work is to demonstrate that the 3D distribution of RI of weak objects can be easily reconstructed by taking advantage of the spatially low coherent illumination. This technique provides speckle-free and enhanced sectioning (a) Intensity

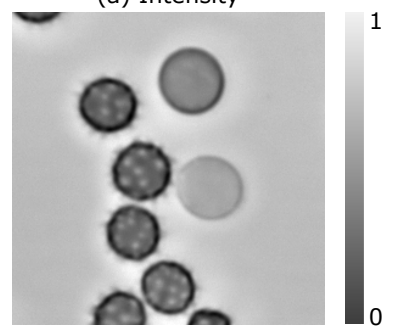

(b) Refractive index $\Delta \mathrm{n}$

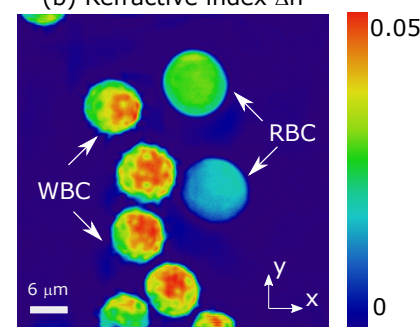

Fig. 4. 2D views within the XY-plane of the (a) intensity and (b) RI distribution $(\Delta n)$ of human blood cells immersed in plasma.

images without needing multiple illuminations resulting in a spatial resolution and a 3D RI reconstruction similar to that obtained by the C-ODT. It is remarkable that only a single intensity stack is needed to reconstruct the 3D-RI. Note also that no staining is required, so this approach is entirely labelfree for the specimen under test. Taking into account the limitations of the first-order Born approximation, the PC-ODT has been tested on diatoms and human blood samples over the range $\Delta n=0.01-0.06$. As a result, it has been proved that the PC-ODT is able to accurately retrieve the RI with a simple experimental setup that can be included in any commercial microscope.

\section{ACKNOWLEDGMENT}

The Spanish Ministerio de Economía y Competitividad is acknowledged for funding the project TEC2014-57394-P.

\section{REFERENCES}

[1] Y. Cotte, F. Toy, P. Jourdain et al., "Marker-free phase nanoscopy," Nat. Photonics, vol. 7, no. 2, pp. 113-117, 2013.

[2] J. Lim, K. Lee, K. H. Jin et al., "Comparative study of iterative reconstruction algorithms for missing cone problems in optical diffraction tomography." Opt. Express, vol. 23, no. 13, pp. 16933-48, 2015.

[3] J. M. Soto, J. A. Rodrigo, and T. Alieva, "Label-free quantitative 3d tomographic imaging for partially coherent light microscopy," Opt. Express, vol. 25, no. 14, pp. 15 699-15 712, Jul 2017.

[4] N. Streibl, "Three-dimensional imaging by a microscope," J. Opt. Soc. Am. A, vol. 2, no. 2, pp. 121-127, Feb 1985.

[5] Y. Bao and T. K. Gaylord, "Quantitative phase imaging method based on an analytical nonparaxial partially coherent phase optical transfer function," J. Opt. Soc. Am. A, vol. 33, no. 11, p. 2125, 2016.

[6] J. B. Sibarita, "Deconvolution microscopy," Adv. Biochem. Eng. Biotechnol., vol. 95, pp. 201-243, 2005.

[7] J. A. Rodrigo and T. Alieva, "Rapid quantitative phase imaging for partially coherent light microscopy," Opt. Express, vol. 22, no. 11, p. 13472, 2014.

[8] S. Yamanaka, R. Yano, H. Usami et al., "Optical properties of diatom silica frustule with special reference to blue light," J. Appl. Phys, vol. 103, no. 7, p. 074701, 2008.

[9] J. Schindelin, I. Arganda-Carreras, E. Frise et al., "Fiji: an open-source platform for biological-image analysis," Nat. Methods, vol. 9, no. 7, pp. 676-682, 2012.

[10] Y. Park, M. Diez-Silva, G. Popescu et al., "Refractive index maps and membrane dynamics of human red blood cells parasitized by plasmodium falciparum," Proc. Natl. Acad. Sci., vol. 105, no. 37, pp. 13730-13735, 2008

[11] T. Debenest, J. Silvestre, M. Coste et al., "Herbicide effects on freshwater benthic diatoms: induction of nucleus alterations and silica cell wall abnormalities," Aquatic Toxicology, vol. 88, no. 1, pp. 88-94, 2008. 